\title{
NON-LINEAR CHARACTERIZATION OF MEMORYLESS SSP AMPLIFIERS
}

\author{
N. T. ALI* \\ Etisalat College of Engineering, Sharjah, UAE
}

(Received 1 February 2002; In final form 31 March 2002)

\begin{abstract}
This paper describes a testbed, which is able to generate all of the standard test signals used for characterising nonlinear amplifiers. The testbed is shown to be sufficiently linear to avoid any possible masking of the non-linear effects of the amplifier under test. All the standard tests: single-carrier, two-carrier, multi-carrier and NPR are applied to a laboratory amplifier and their results found to be in reasonably good agreement with the most popular prediction techniques. The merits of the individual test have been highlighted.
\end{abstract}

Keywords: Amplifier, non-linear, characterization and memory

\section{INTRODUCTION}

Orthogonal Frequency Division Multiplex (OFDM) or multi-carrier transmission schemes have been proposed for many applications where high-speed data must be transmitted over a dispersive (delay spread) channel. The major drawback of multi-carrier schemes is their high peak to mean envelope power ratio, which is equal to the number of carriers. In principle, to transmit an $\mathrm{N}$-carrier signal with an average power of $1 \mathrm{~W}$, an amplifier, which is linear up to $\mathrm{N}$ Watts, is required. The DC to RF power conversion efficiency of such an amplifier is unacceptably low for most applications. On the other hand, if the peaks of the signal are allowed to drive the amplifier into saturation, the intermodulation distortion generated gives rise to high levels of out-of-band radiation. This is a particular problem in the Wireless LAN ad-hoc network, where a node may be trying to receive a signal on one channel from a distant transmitter while a nearby node is transmitting on the adjacent channel. It is therefore particularly important to be able to predict the intermodulation distortion generated by non-linear amplification.

There is no generally accepted industry standard method for characterising non-linear amplifiers. Several techniques are described in the literature [1-8] and all have advantages and disadvantages. Some companies favour one technique and some, another. The four main techniques are described in Section 2. The first two rely on simple measurements using only one or two carriers, and predict the performance with more carriers by using a mathematical model. Such models rely on the assumption that the amplifier is memoryless. The other two techniques use a more direct approach applying a multicarrier test signal or a wideband

\footnotetext{
* E-mail: ntali@ece.ac.ae
} 
noise signal to simulate the effects of an infinite number of carriers, and then scaling the results to other numbers of carriers. These techniques do not rely on the memoryless assumption.

Section 3 describes a testbed based around the Hewlett Packard HP8770 Arbitrary Waveform Synthesiser. This is able to generate any of the standard test signals, with one, two or many carriers or a noise signal, by pre-loading its memory at low speed with a series of time samples generated off-line by computer, and reading out the memory to the digital to analogue converter (DAC) at $125 \mathrm{MHz}$ sampling rate to generate a signal of up to $62.5 \mathrm{MHz}$ bandwidth. The resulting signal is converted to the HIPERLAN frequency band of $5.2 \mathrm{GHz}$ by a highly linear triple up-converter. Intermoduloation components in the $5.2 \mathrm{GHz}$ test signal are at least $60 \mathrm{~dB}$ below the two-carrier signal level, allowing accurate measurements of the distortion generated by the amplifier under test over a wide dynamic range.

In Section 4 the performance predicted by the mathematical models of the single and twocarrier tests are compared to the direct measured results with four or ten carriers and the Noise Power Ratio (NPR) test. In Section 5 the conclusions are summarised.

\section{THEORY}

The intermodulation performance of non-linear amplifiers can be predicted from the following tests. The first two are model-based approaches that both rely on the underlying assumption that the amplifier is memoryless. This means that the intermodulation distortion at time ' $t$ ' depends only on the signal amplitude at time ' $t$ ' and not on its value at any previous or later time. In practical terms this translates into a requirement that the bandwidth of the amplifier should be substantially wider than the signal it is being used to amplify, i.e. any filtering required is performed separately and does not rely on the frequency response of the amplifier itself. This requirement is not difficult to meet for most practical applications, and in particular for the Wireless LAN application of interest to the authors. The other two tests are direct measurements of amplifier performance with a representative multi-carrier signal, and therefore equally valid whether the amplifier is memoryless or not. The main objective of this paper is to compare the performance measured by the direct methods with that predicted by the model-based methods for an amplifier, which does satisfy the memoryless requirement.

\subsection{Single-Carrier Test}

In this test the output power $v s$. input power (AM/AM) and phase shift vs. input power $(\mathrm{AM} / \mathrm{PM})$ measurements are made and the curves fitted by a suitable model $[2,3]$. The saturation curves for other numbers of carriers are then found by applying the statistical distribution of input signal envelope to the single carrier model for various average power levels, and averaging over the output power distribution. The input signal envelope distribution converges to a Rayleigh distribution of course, for large numbers of carriers. Intermodulation performance may then be predicted by the Inter-Modulation Analysis (IMAL) simulation software [4] generated by the European Space Agency, or by Bessel function analysis [5].

\subsection{Two-Carrier Test with Intercept Analysis}

This test relies on the Taylor Series model of amplifier non-linearity. With two carriers at frequencies $f_{1}$ and $f_{2}$, third order products can occur only at $2 f_{1}-f_{2}$ and $2 f_{2}-f_{1}$. These increase in power at $3 \mathrm{~dB}$ per $\mathrm{dB}$ increase in input power. Fifth order products can occur only at $3 f_{1}-2 f_{2}$, $2 f_{1}-f_{2}, 2 f_{2}-f_{1}$ and $3 f_{2}-2 f_{1}$. These increase at $5 \mathrm{~dB}$ per $\mathrm{dB}$ increase in input power. Seventh 
and higher order products behave similarly. At high input power levels it is not possible to distinguish the third order products from the fifth and higher. However, because these fall off more rapidly as the input power is reduced, there is a range of power levels in which the third order products can be clearly distinguished at $2 f_{1}-f_{2}$ and $2 f_{2}-f_{1}$. By fitting a straight line of gradient $3 \mathrm{~dB} / \mathrm{dB}$ to these points, and projecting it to the point where it intercepts the linear term, a direct estimate of the third order coefficient of the Taylor series can be made. Similarly, there is a range of power levels where the fifth order products at $3 f_{1}-2 f_{2}$ and $3 f_{2}-2 f_{1}$ are significant and the seventh and higher order products are not. By fitting a straight line of gradient $5 \mathrm{~dB} / \mathrm{dB}$ to these points a direct estimate of the fifth order coefficient of the Taylor series can be made. Once the Taylor series expansion of the amplifier transfer characteristic is derived it may be applied directly to predict the performance with any chosen number of carriers.

\subsection{Multi-Carrier Test}

The most straightforward test is to make a direct measurement of the signal to intermodulation ratio with exactly the same number of carriers as are to be used in the practical system. This is generally not known to the amplifier designers in advance. Bond and Meyer [6] have shown that where third order products are dominant, performance with any number of carriers greater than 7 can be predicted from a test with any other number of carriers greater than 7 , by simple scaling of the carrier power. Where fifth and seventh order products must be taken into account, a 15-carrier test is sufficient to predict performance for numbers of carriers greater than 15 . The multi-carrier test generally uses ten or more carriers on a uniformly spaced raster with a measurement slot provided by leaving one carrier vacant near the centre of the spectrum.

Multi-carrier tests have been proposed using fewer carriers than this, e.g. four, three or even two. In these cases, the performance with different numbers of carriers cannot be predicted directly by scaling the carrier power and a "rule-of-thumb" which is based on the assumption of a third order model must be used instead.

\subsection{Noise Power Ratio (NPR)}

In this test a white noise source is passed through a band-stop filter to produce a deep notch in the spectrum. This effectively simulates an infinite number of carriers and the intermodulation noise power spectral density $(\mathrm{mW} / \mathrm{Hz})$ may be measured directly in the notch frequency at the output of the amplifier. By the same arguments mentioned in 2.3 above, the performance for numbers of carriers down to 7 or 15 can be predicted from this test by scaling the carrier power, provided higher order products above third or seventh respectively can be ignored. One of the main problems with the NPR test in the past has been the realisation of suitable deep notch filters. The transition from pass to stop-band in conventional filters means that maximum dynamic range is only achieved at the very centre of the notch. An alternative technique is to generate the noise spectrum digitally and produce the notch by forcing some of the points in its Fourier spectrum to zero. The test signal is then produced by the Inverse Fourier Transform giving a very clean, square sided notch. Strictly speaking, this signal actually represents a finite number of carriers equal to the size of the Fourier Transform, and coherent over the transform block length. Care must therefore be taken in interpreting the results.

\section{EXPERIMENTAL SET-UP}

Figure 1 is a block diagram of the experimental set-up. The Arbitrary Waveform Synthesiser (HP8770) generates its output waveform by digital to analogue conversion (DAC) of the con- 


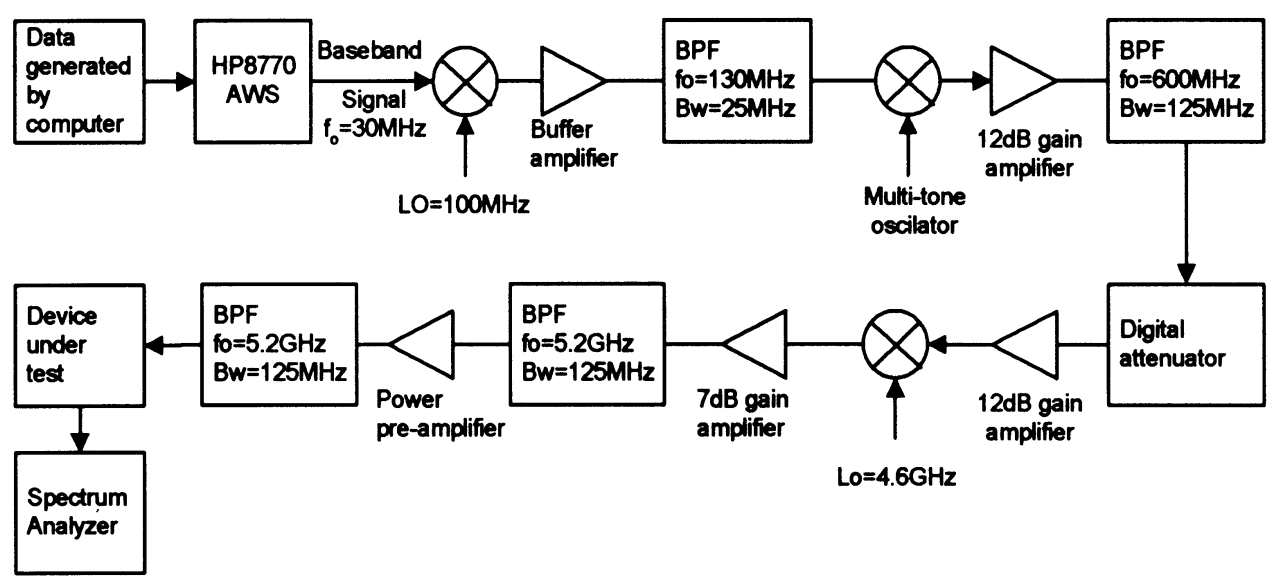

FIGURE 1 Block diagram of the experimental set-up.

tents of its memory at $125 \mathrm{MHz}$ sampling rate. The memory is pre-loaded with samples generated off-line, representing any desired waveform, from the computer. The Nyquist bandwidth is $62.5 \mathrm{MHz}$, and therefore the test waveforms were generated with a centre frequency of $31.25 \mathrm{MHz}$. A highly linear triple up-converter was built to raise the test signals' centre frequency to $130 \mathrm{MHz}, 600 \mathrm{MHz}$, and finally $5.2 \mathrm{GHz}$. The exact output frequency was specified in a Radio Transmission license granted for HIPERLAN propagation testing, and was used so that the equipment could serve a dual purpose.

Test waveforms for the single-carrier, two-carrier, four-carrier, ten-carrier and NPR tests were generated. In the case of the two-carrier the intermodulation products generated by the test set-up are at least $60 \mathrm{~dB}$ below the level of the carriers. This means the dynamic range of this test is $60 \mathrm{~dB}$ and the measured third and higher order intermodulation levels can be used with confidence down to $-60 \mathrm{~dB}$ in calculating the intercept points.

However, the dynamic range of the other test signals, with more than 2 carriers, becomes progressively smaller as the total available power is shared among more carriers, but this is not an issue as these tests are used for direct measurement of the intermodulation power, rather than estimating the parameters of the Taylor Series model. Provided the intermodulation noise density can be measured at levels well below the operating signal to noise ratio it is not necessary to extend the dynamic range further.

\section{RESULTS}

Input/output Backoffs are defined in this paper as the input/output powers normalized to the single carrier saturation point, i.e. input backoff $(\mathrm{dB})=10 \log$ (total input power/ 1 carr. input sat.). A background review of the theory is summurized in the Appendix.

The amplifier transfer characteristics measured with the single-carrier, two-carrier, fourcarrier, ten-carrier and NPR test signals are shown in Figure 2a. The AM/PM conversion characteristic measured with the single carrier test signal is shown in Figure $2 \mathrm{~b}$. The transfer characteristics for one, two, four and ten carriers and for the NPR signal were calculated from the single carrier results using IMAL. The transfer characteristics for one and two carriers were also calculated using Bessel function analysis, by Shimbo [5]. The expected carrier to intermodulation ratio was also calculated for two, four and ten carriers and NPR using IMAL and Taylor methods. 

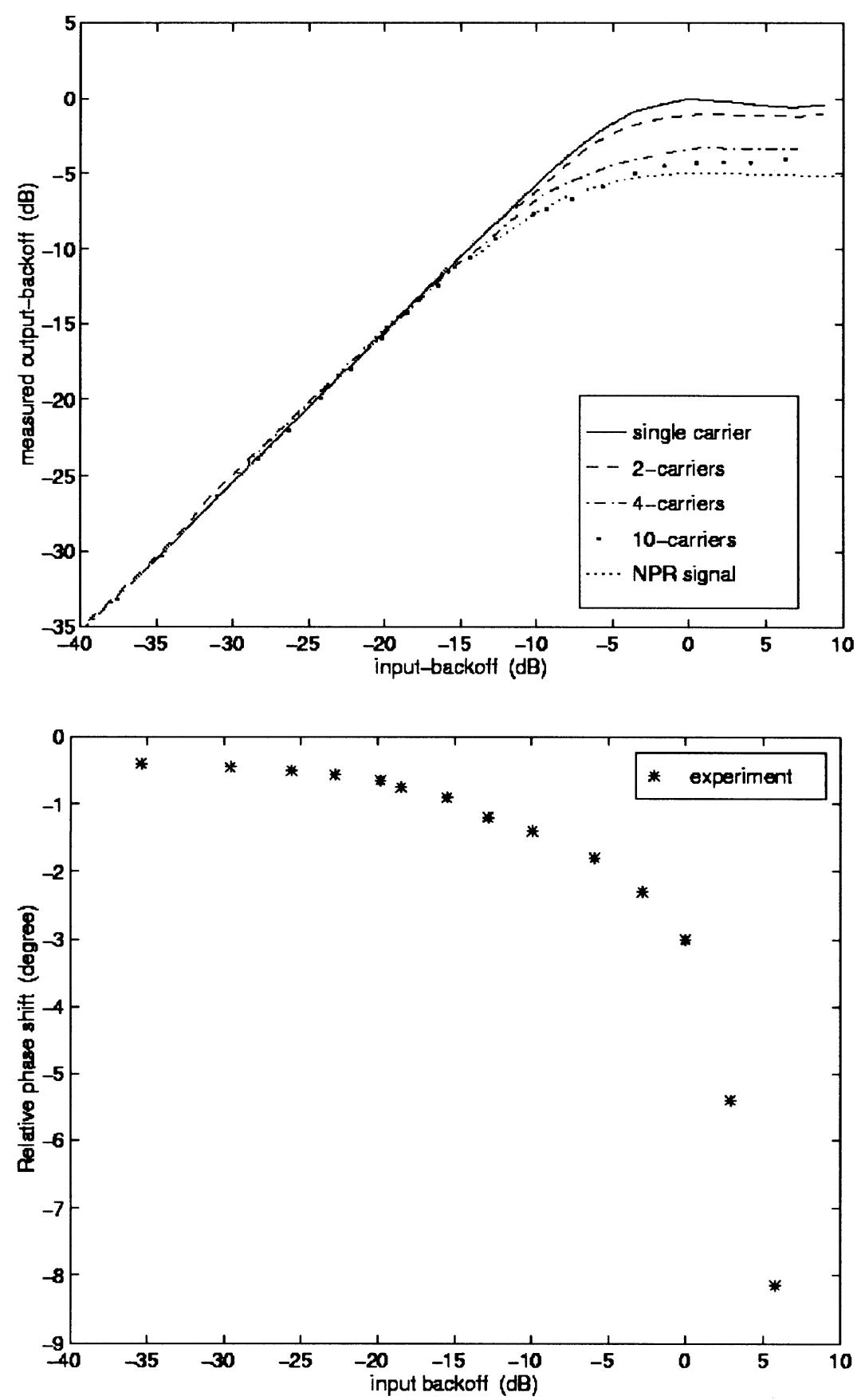

FIGURE 2 (a) HPA output power and (b) output phase vs. input power.

The results of the two-carrier test and intercept analysis are shown in Figure 5. These results were used to generate the coefficients of the Taylor Series model, which was then used to predict the transfer characteristic and carrier to interference ratio for four and ten carriers and the NPR, as well as the transfer characteristic for the single carrier case. 


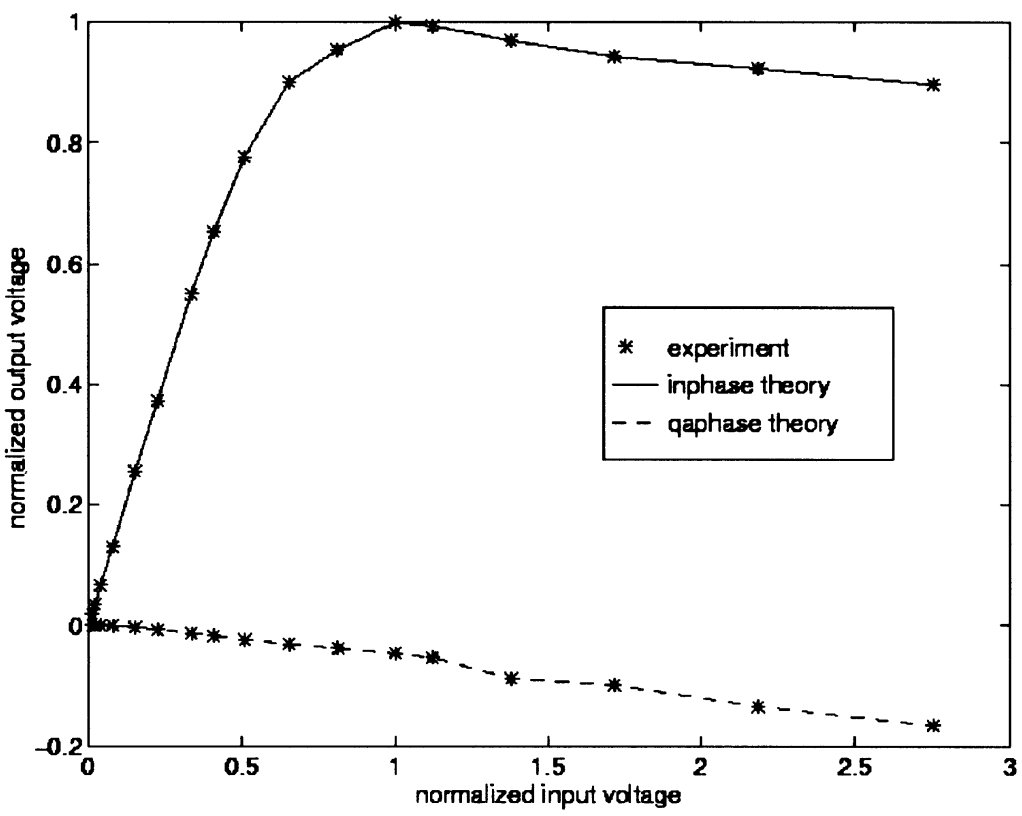

FIGURE 3 Quadrature model for single carrier measurements.

Figure 2a shows the input-output power transfer curves for different number of carriers. As expected, at relatively low input power, the output signal would respond linearly to the input signal and with constant gain, regardless of the number of carriers. This is different near or at saturation, as some of the output power will be shared by the intermodulation com-

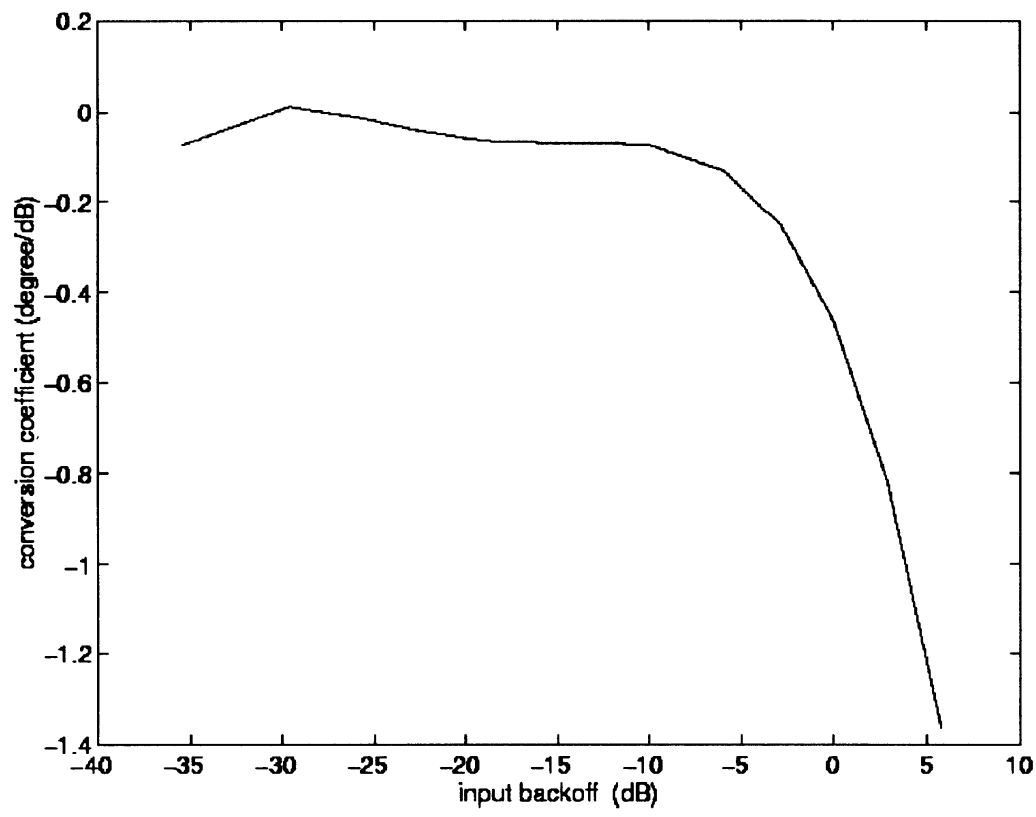

FIGURE 4 Calculated conversion coefficient $K v s$. input-backoff. 


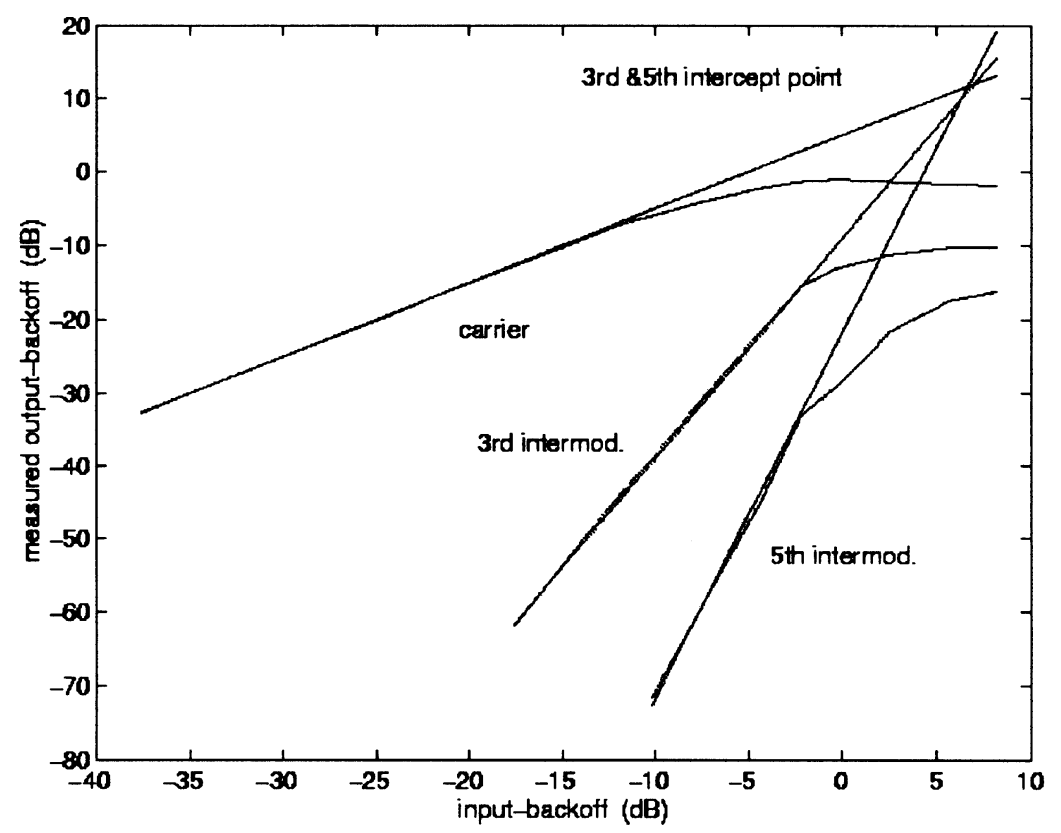

FIGURE 5 Definition of intercept points for the 2-carrier case.

ponents. The amount of power lost would intensify with the number of carriers resulting in an increasing reduction in the output power contained in the fundemental carriers.

Figure $6 \mathrm{a}$ shows the single carrier measurements, fitted by IMAL software and Bessel function, as well as predicted by Taylor series from the two carrier measurements. Two

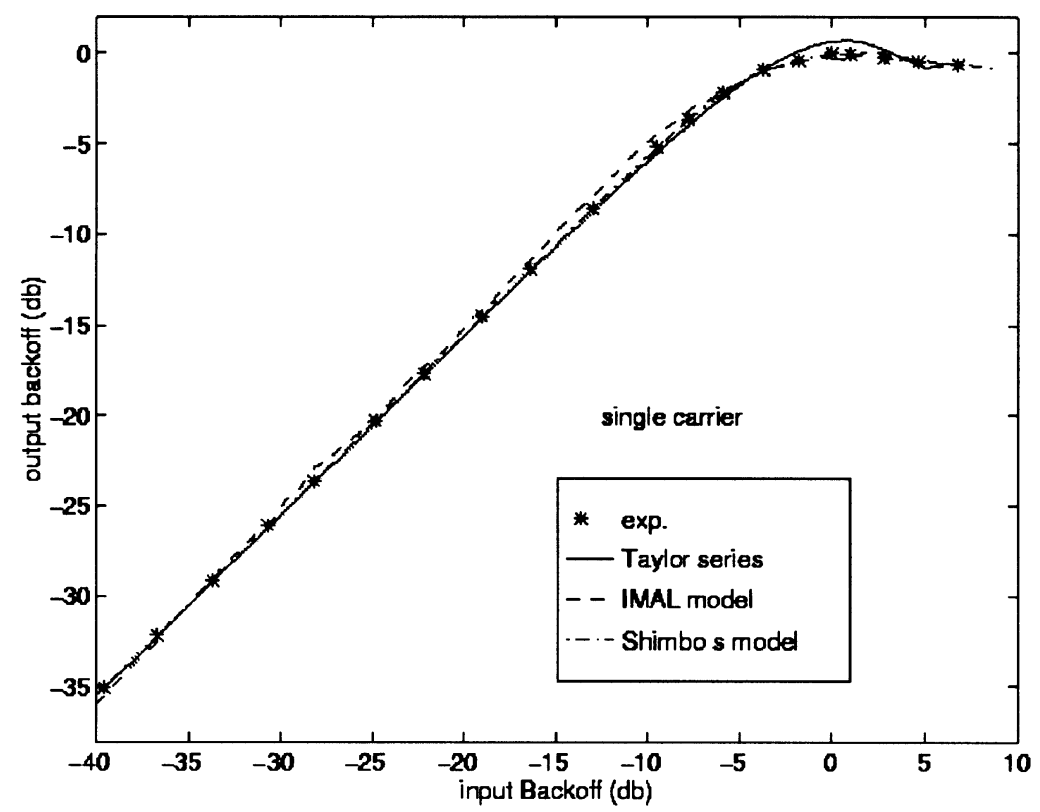

FIGURE 6 Correlation between experiment and theory for different number of carriers. 

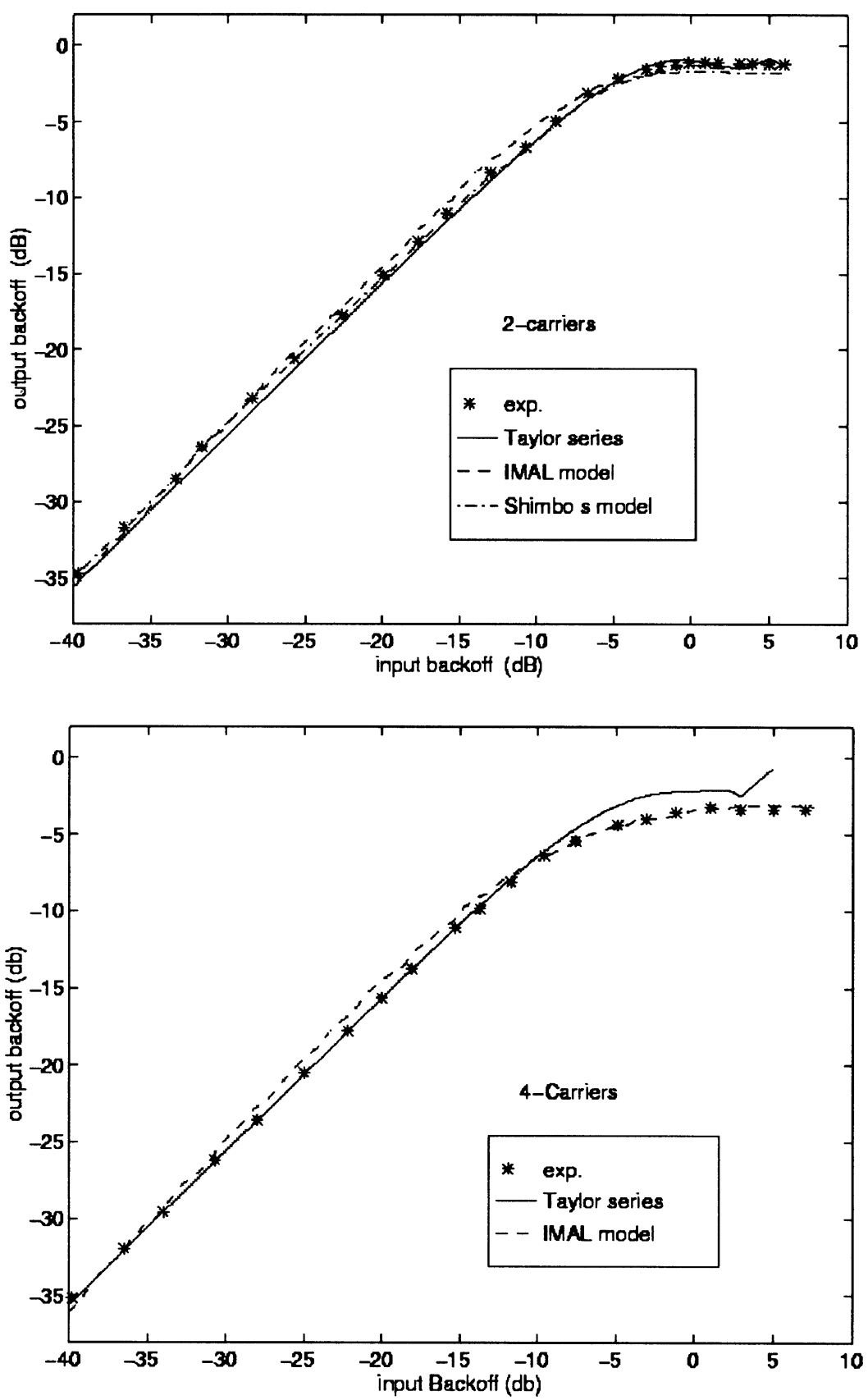

FIGURE 6 (Continued)

coefficients (like Saleh's) or four coefficients (like Franchi's) formulas could either be used to obtain the coefficients necessary for IMAL software. The mathematical expressions could still be used to either model, the AM/AM and AM/PM distortions separately, or both combined in a quadrature format, Figure 3. Either way, the accuracy of any formula will be affected greatly by the number of coefficients required to fit a certain curve, in other words, the harder the amplifier is driven into saturation the more coefficient are required. 

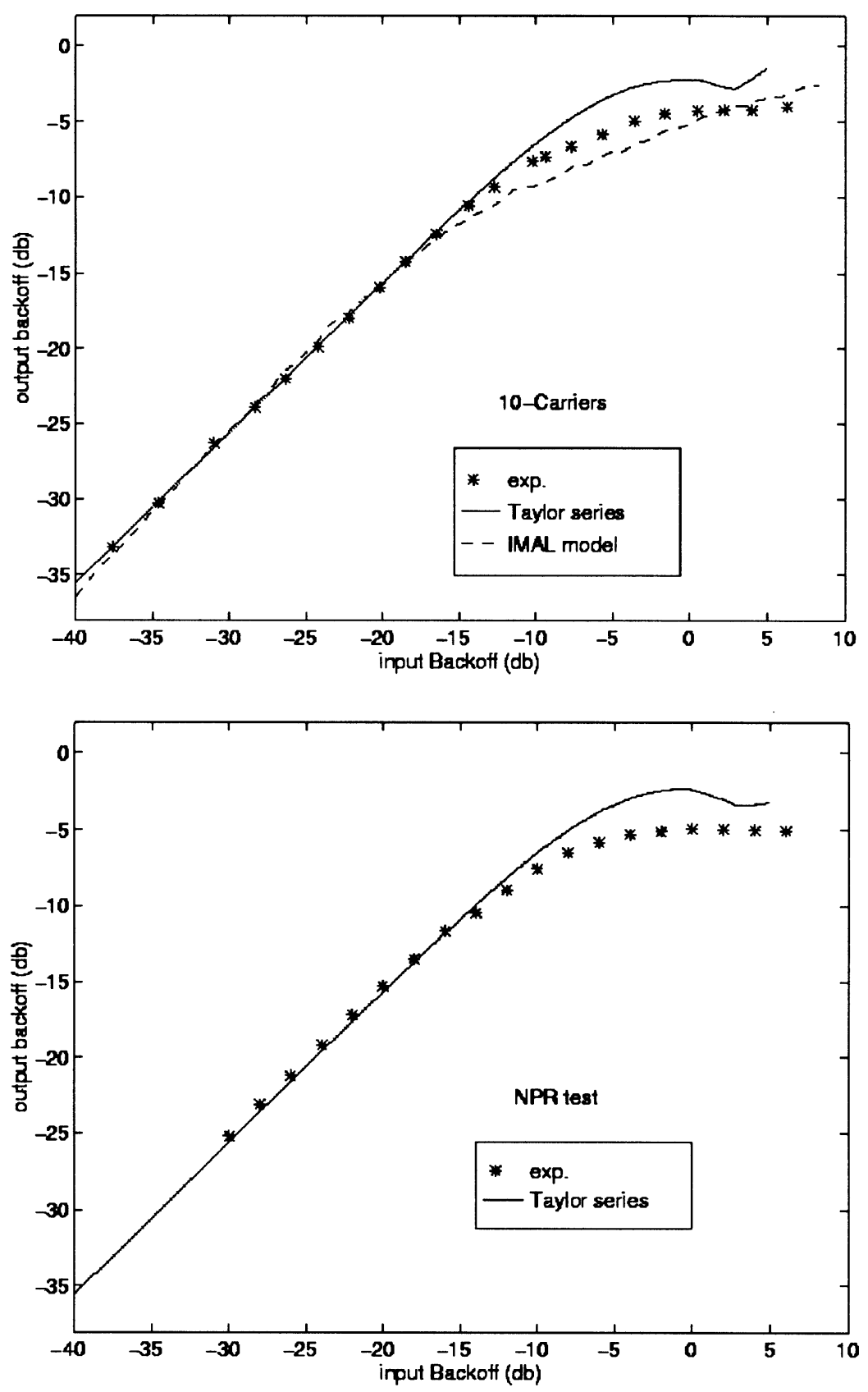

FIGURE 6 (Continued)

Figure $6 \mathrm{a}$ also shows Taylor series prediction from the two carrier measurements. It gives a perfect fit up to about $3 \mathrm{~dB}$ backoff, where after it over shoots the saturation region by about $0.5 \mathrm{~dB}$ max. This, again, can be attributed to the number of terms used in the power series.

Figures $6 \mathrm{~b}$ and $7 \mathrm{a}$ depict the transfer characteristics of the amplifier under the two-carrier test. Only four coefficients were used in Taylor series fittings. They were obtained from the fundamental, the $3 \mathrm{rd}$, the 5th, and the 7 th intermodulation components (Fig. 5). It was very 

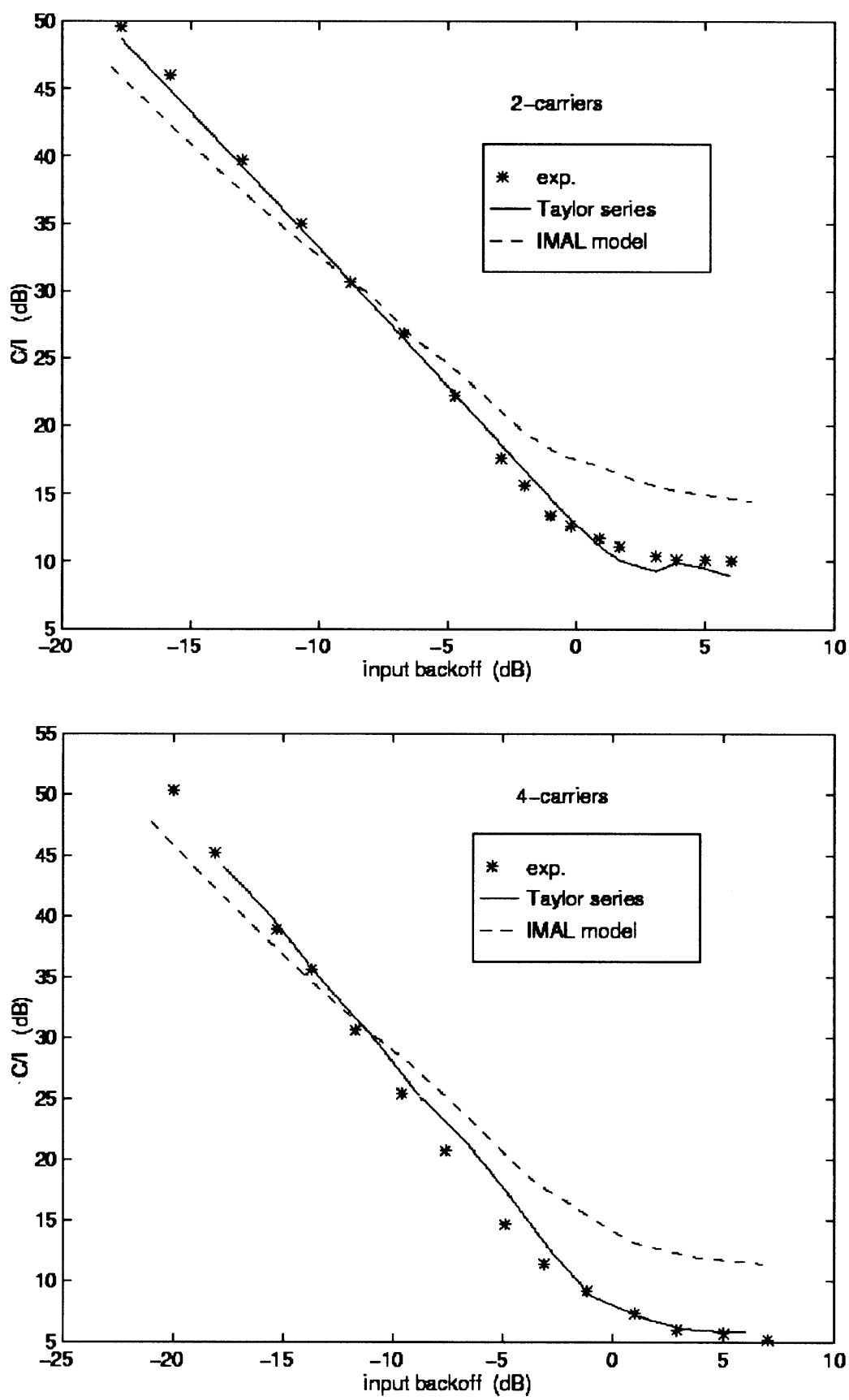

FIGURE 7 C/I ratio for different number of carriers.

difficult to measure any higher intermod level. However, this was found to give reasonable fitting and prediction under different number of carriers. The expected results were obtained from Bessel function and IMAL software. Bessel gives a very good approximation, as the quadrature representation was used which includes the effect of AM/PM distortion. IMAL, however, depends on many parameters, such as the formula used to distort the envelope, see the Appendix, and whether the phase distortion is severe or not. 

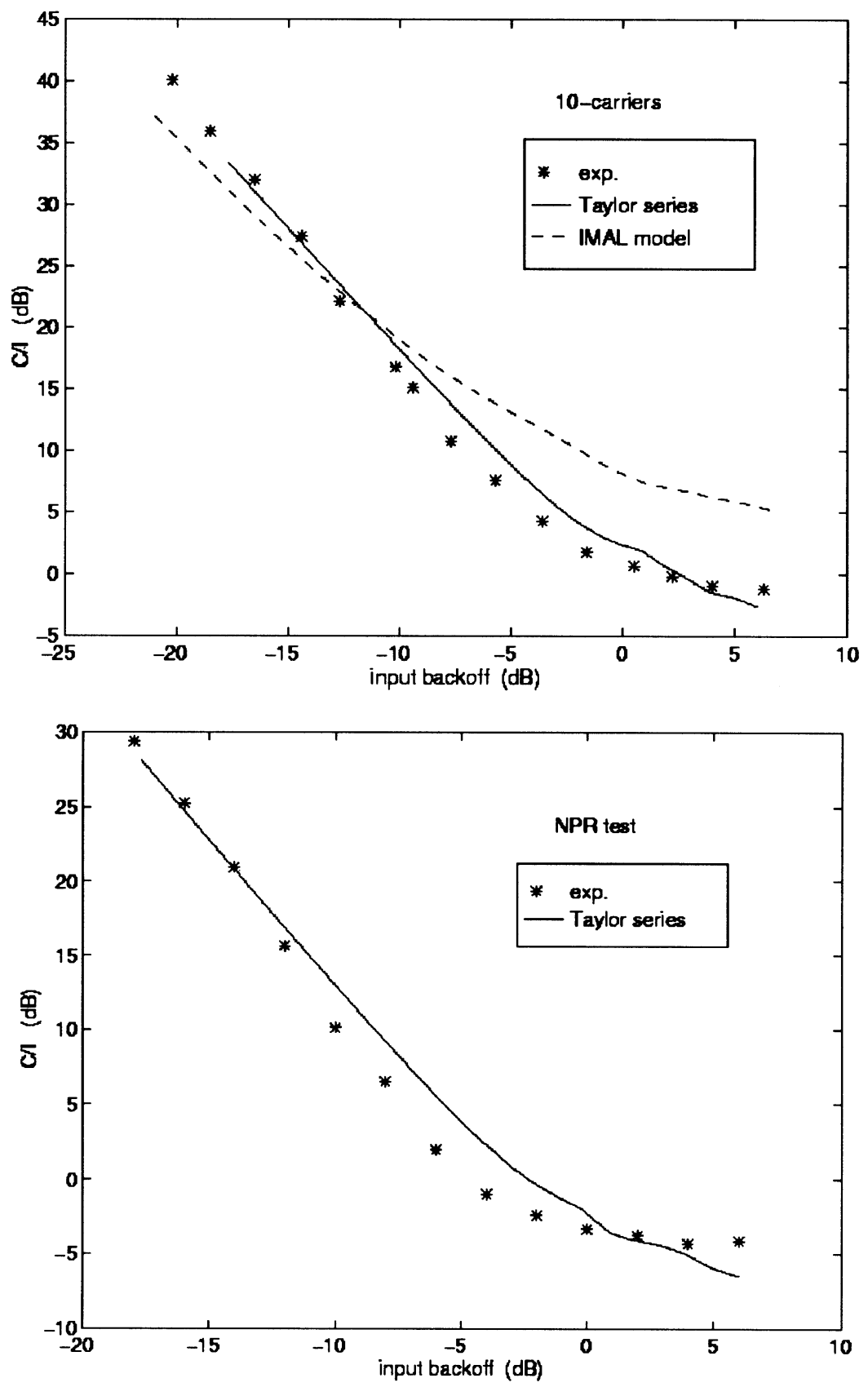

FIGURE 7 (Continued)

Figures $6 \mathrm{c}-\mathrm{e}$ and $7 \mathrm{~b}-\mathrm{d}$ give the 4, and the 10-carriers measurements compared against Taylor and IMAL calculations as well as Taylor prediction for a very high number of carriers manifested in the NPR test. As can be seen Taylor expectations is increasingly worsen as the number of carriers increases. This can be attributed to the following.

When number of carriers is higher than two, it becomes very difficult to measure the level of the fundamental carrier as some of the intermodulation products would fall in the carrier 
position. Equation (12), of the Appendix, gives the level of the fundamental carriers only, without the effect of the intermodulations generated through both, amplitude and phase distortions. For example, for the 10-carriers case, although they are of equal amplitude at the input, their levels at the output are different. This is a direct result of the number of the intermodulation components falling in with a carrier and the way they add up, i.e. on either voltage or power basis.

\section{CONCLUSION}

The single-carrier and 2-carrier with intercept analysis test methods are simple, quick and popular, but their accuracy dependent on the mathematical models available to predict the performance under higher number of carriers. On the other hand, multi-carriers and NPR test methods can produce more accurate results as they manifest a realistic load conditions.

In this paper Taylor series and IMAL, softwares were used to simulate the behaviour of the SSP amplifier under different conditions. Both methods were found to be very useful and accurate tools for extrapolating the single or two carrier measurements to higher number of carriers.

Taylor series is more suitable for straightforward analysis, i.e. for unmodulated sub-carriers and without any type of noise. It also gives a better and clear indication of the location of the carriers and their intermodulation components in the spectrum. Comparing Eqs. (5) and (12) of the Appendix, it can be seen that Taylor series can cope with high number of carriers a lot better than Bessel series.

However, Shimbo's method (Bessel series) is more general, where it covers the effect of noise and phase modulated signals. In addition, it was found to be relatively more accurate when was used with a very limited number of carriers.

\section{References}

[1] Stette, G. (1987). Large/small carrier performance of nonlinear devices. International Journal of Satellite Communications, 5, 291-294.

[2] Adel, A. M. Saleh, (1987). Frequency-independent and frequency-dependent nonlinear models of TWT amplifiers. Proceeding of the IEEE, 5, 291-294.

[3] Franchi, A., Colzi, E., Elia, C. and Harris, R. A. (1995). Performance of COFDM for satellite digital audio broadcasting. International Journal of Satellite Communications, 13, 229-242.

[4] European space research and technology centre. (1990). Software for analysis of power amplifiers.

[5] Shimbo, O. (1971). Effect of intermodulation, AM-PM conversion, and additive noise in multicarrier TWT system. Proceeding of the IEEE, 59(2).

[6] Bond, F. E. and Meyer, H. F. (1970). Intermodulation effects in limiter amplifier repeaters. IEEE Transactions on Communication Technology, 18(2).

[7] Kavehrad, M. (1981). Multiple FM/FDM carriers through nonlinear amplifier. IEEE Transactions on Communications, 29(5).

[8] Westcott, R. J. (1967). Investigation of multiple FM/FDM carriers through a satellite TWT operating near to saturation. Proc. Inst. Elec. Engineering, 114(6).

\section{APPENDIX}

\section{A.1 Single Carrier Method}

\section{A.1.1 IMAL Model}

Figure 8 shows the schematic diagram of the computer model used by IMAL. The input, which could be either one signal or multi-signals, is usually given in the frequency domain. 


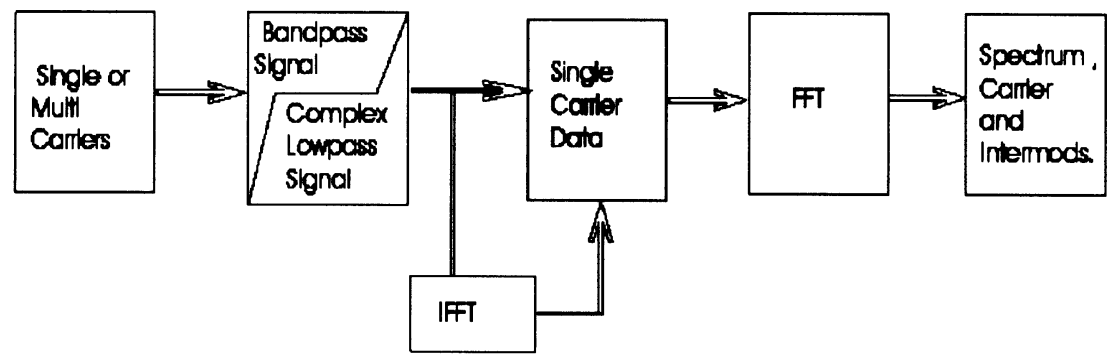

FIGURE 8 Block diagram of IMAL software.

Each spectral component was given an initial random phase value and by performing Inverse Fast Fourier Transform (IFFT) the spectrum was then converted to the time domain.

Since only the envelope carries the information and not the carriers, the distortion only to the envelope was considered. In addition, as only the intermodulation products falling in the transmission band are of interest, the band pass signal is down-converted to a complex lowpass form and then, distorted by the measured AM/AM and AM/PM data.

A straightforward linear interpolation routine was used to distort the input signal point by point. This can be performed easily by using either Saleh formulas [2] or a more flexible one like Franchi [3].

Following the interpolation process, the data was then transferred back into frequency domain (FFT), in order to evaluate the output spectrum, which includes carriers and intermodulation products.

\section{A.1.2 Shimbo's Model}

Consider the case of $n$ equispaced and equal unmodulated sub-carriers, the input to the nonlinear device is

$$
e_{i}(t)=R\left\{\sum_{l=1}^{n} A e^{j\left(w_{o} t+w_{l}+\theta_{l}(t)\right)}\right\}
$$

where $w_{o}, w_{l}$, respectively are the carrier and sub-carrier angular frequencies and $\theta_{l}$ is the relative phase shift.

Shimbo was concerned with the non-linear distortion caused by the envelope of a bandpass signal. Therefore if Eq. (1) can be re-written as

$$
e_{i}(t)=R\left\{\rho(t) e^{j\left(w_{o} t+\Phi(t)\right)}\right\}
$$

Then, the output could take this form,

$$
e_{o}(t)=R\left\{g\{\rho(t)\} e^{j\left(w_{o} t+\Theta(t)+h\{\rho(t)\}\right)}\right\}
$$

$\rho(t)$ is the amplitude of the Rf signal, $g\{\rho(t)\}$ and $h\{\rho(t)\}$ are the output amplitude and phase functions, respectively.

Shimbo [6] has shown that, and in the absence of any kind of noise, the output can also be expressed as

$$
e_{o}(t)=R\left\{\sum_{k_{1}=-\infty}^{\infty} \sum_{k_{2}=-\infty}^{\infty} \cdots \sum_{k_{n}=-\infty}^{\infty} \exp \left[j \sum_{l=1}^{n} k_{l} \theta_{l}(t)\right] M\left(k_{1}, k_{2}, \ldots, k_{n}\right)\right\}
$$


$k_{1}+k_{2}+\cdots+k_{n}=1$ are a set of integers used to describe the order of any carrier and intermodulation product. While $M\left(k_{1}, k_{2}, \ldots, k_{n}\right)$ is the complex amplitude of each spectral component of the output signal, given by

$$
M\left(k_{1}, k_{2}, \ldots, k_{n}\right)=\sum_{s=1}^{m} b_{s} \prod_{l=1}^{n} J_{k l}\left(\alpha_{s} A_{l}\right)
$$

From Eq. (5) the expression for the single carrier case can be obtained $(n=1)$,

$$
M(1,0,0, \ldots, 0)=\sum_{s=1}^{l} b_{s} J_{l}\left(\alpha_{s} A\right)
$$

In Eqs. (5) and (6) $J_{k l}\left(\alpha_{S} A_{l}\right)$ is Bessel function of the first kind of order $k_{l}$ and $b_{s}$ are coefficients which can be calculated from the single carrier measurement, as shown in the section, by means of least square error. Using Bessel model, [6], it was shown that in order to derive the characteristics of the non-linear device from the single carrier transfer curve most accurately, the quadrature model, Figure 3, would have to be employed. For the sinusoidal input $\operatorname{Re}\left\{A \cdot e^{j w_{o} t}\right\}$, the single carrier output may be written as,

$$
e_{o}(t)=R\left\{g(A) e^{j w_{o} t+j f(A)}\right\}
$$

The inphase and quadrature components of the complex envelope of the output signal are

$$
\begin{aligned}
& g(A) \cos (f(A))=\sum_{s=1}^{L} b_{s r} J_{1}\left(\alpha_{s} A\right) \\
& g(A) \sin (f(A))=\sum_{s=1}^{L} b_{s q} J_{1}\left(\alpha_{s} A\right)
\end{aligned}
$$

The least square error method was also used to determine the values of $b_{s r}$ and $b_{s q}$ and, consequently, it has been found that optimum results, Figures 6 , were obtained when the number of coefficients equaled the number of pairs of input-output data.

\section{A.2 Two-Carrier Method}

\section{A.2.1 AM-AM Conversion}

For the same case of section A.1.2,

$$
e_{i}(t)=R\left\{\sum_{l=1}^{n} A e^{j\left(w_{o} t+w_{l} t+\theta_{l}\right)}\right\}
$$

The input/output voltage/power relationship, Figure 2, can be approximated by a power series, e.g. Taylor series and if $w_{o} \gg w_{l}$ then only the odd powers in the series are important, therefore, the instantaneous transfer characteristics could take this form6

$$
e_{o}(t)=\sum_{k=1}^{\infty} a_{(2 k-1)} e_{i}^{(2 k-1)}(t)
$$


Although, system impedance was around $50 \Omega$, with total input power of $n A^{2} /(2 * 50)$. The expression obtained from Eqs. (10) and (12), which gives the amplitude of the $n$th output carrier was normalized to $1 \Omega$ for simplification purposes

$$
A_{n}=a_{1} \sqrt{\frac{2 P_{i t}}{n}}\left[1+3 \frac{a_{3}}{a_{1}} \frac{P_{i t}}{n}\left(n-\frac{1}{2}\right)+15 \frac{a_{5}}{a_{1}}\left(\frac{P_{i t}}{n}\right)^{2}\left(n^{2}-\frac{3 n}{2}+\frac{2}{3}\right)+\cdots\right]
$$

For large $n$, the terms within the brackets (known as compression factor) would be approximated to

$$
A_{\infty}=a_{1} \sqrt{\frac{2 P_{i t}}{n}}\left[1+3 \frac{a_{3}}{a_{1}} P_{i t}+15 \frac{a_{5}}{a_{1}}\left(P_{i t}\right)^{3}+\cdots\right]
$$

In addition to the carrier outputs, there are many intermodulation products, the most important of which, i.e. products falls inband, are as follows

1 Products of the form $\left(2 f_{1}-f_{2}\right)$, whose amplitudes are

$$
I_{n}=\frac{3}{4} a_{3}\left(\frac{2 P_{i t}}{n}\right)^{3 / 2} F_{n}\left(P_{i t}\right)
$$

where

$$
\begin{aligned}
F_{n}\left(P_{i t}\right)=\{ & 1+\frac{2 a_{5}}{3 a_{3}} \frac{P_{i t}}{n}[12.5+15(n-2)]+105 \frac{a_{7}}{a_{3}}\left(\frac{P_{i t}}{n}\right)^{2} \\
& \left.\times\left[(n-2)(n-3)+\frac{13}{6}(n-2)+\frac{7}{12}\right]+\cdots\right\}
\end{aligned}
$$

2 Products of the form $\left(f_{1}+f_{2}-f_{3}\right)$, whose amplitudes are

$$
I_{n}^{\prime}=\frac{3}{2} a_{3}\left(\frac{2 P_{i t}}{n}\right)^{3 / 2} F_{n}^{\prime}\left(P_{i t}\right)
$$

where

$$
\begin{aligned}
F_{n}\left(P_{i t}\right)=\{1 & +10 \frac{a_{5}}{a_{3}} \frac{P_{i t}}{n}\left[\frac{3}{2}+(n-3)\right]+210 \frac{a_{7}}{a_{3}}\left(\frac{P_{i t}}{n}\right)^{2} \\
& \left.\times\left[1+\frac{7}{4}(n-3)+\frac{1}{2}(n-3)(n-4)\right]+\cdots\right\} .
\end{aligned}
$$

According to Westcott [4], the number of intermodulation products falling in the cetre of the signal spectrum can be calculated; for the $\left(2 f_{2}-f_{1}\right)$ type

$$
v_{D n}=\frac{n-2}{2}
$$

and for the $\left(f_{1}+f_{2}-f_{3}\right)$ type

$$
v_{D n}^{\prime}=\frac{(n-2)(3 n-4)}{8}
$$


From the preceding the carrier to the total intermodulation ratio can be calculated as shown below

$$
\left(\frac{C}{I_{\mathrm{AM} / \mathrm{AM}}}\right)_{n}=\frac{A_{n}^{2}}{v_{D n} I_{n}+v_{D n}^{\prime} I_{n}^{\prime}}=\frac{4 n^{2}\left(a_{1} / a_{3}\right)^{2} F_{n o}^{2}}{9 P_{i}^{2}\left(v_{D n} F_{n}^{2}+4 v_{D n}^{\prime} F_{n}^{\prime 2}\right)}
$$

\section{A.2.2 AM-PM Conversion}

Previous research has shown that the AM/PM distortion would result in intermodulation components of the same form as that resulted from the AM/AM with the same frequency locations, i.e. $\left(2 f_{1}-f_{2}\right),\left(f_{1}+f_{2}-f_{3}\right), \ldots$ etc. Following the same procedure, [4] has shown that the AM-PM conversion results in a carrier to intermodulation ratio of

$$
\left(\frac{C}{I_{\mathrm{AM} / \mathrm{PM}}}\right)_{n}=\frac{n^{2}}{(0.1516 K)^{2}} \frac{8}{3(n-2)(n-1)}
$$

and as $n$ increases, the expression will converge to

$$
\left(\frac{C}{I_{\mathrm{AM} / \mathrm{PM}}}\right)_{n}=\frac{116}{K^{2}}
$$

The AM-PM conversion coefficient $K($ degree/dB) can be determined from the single carrier data by calculating the slope of output phase shift against input power-curve, as shown in Figure 4. $K$ values can then be used for any number of carriers. In order to incorporate the two types of distortion in one expression, it was assumed that all intermod components are noncoherent (not completely true), therefore they can be added on power bases

$$
\left(\frac{C}{I_{\mathrm{tot}}}\right)=\frac{C}{I_{\mathrm{AM} / \mathrm{AM}}+I_{\mathrm{AM} / \mathrm{PM}}} .
$$



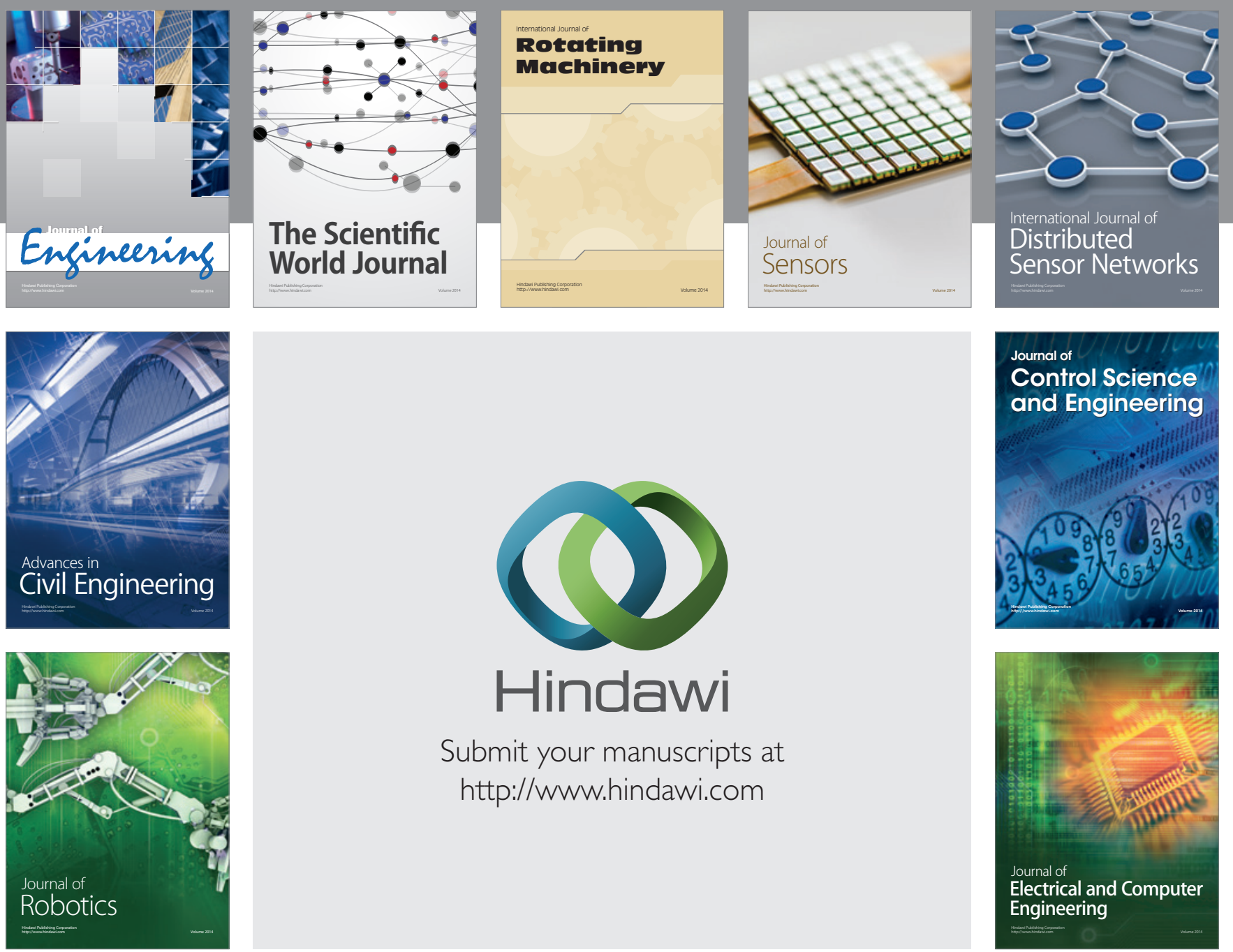

Submit your manuscripts at

http://www.hindawi.com
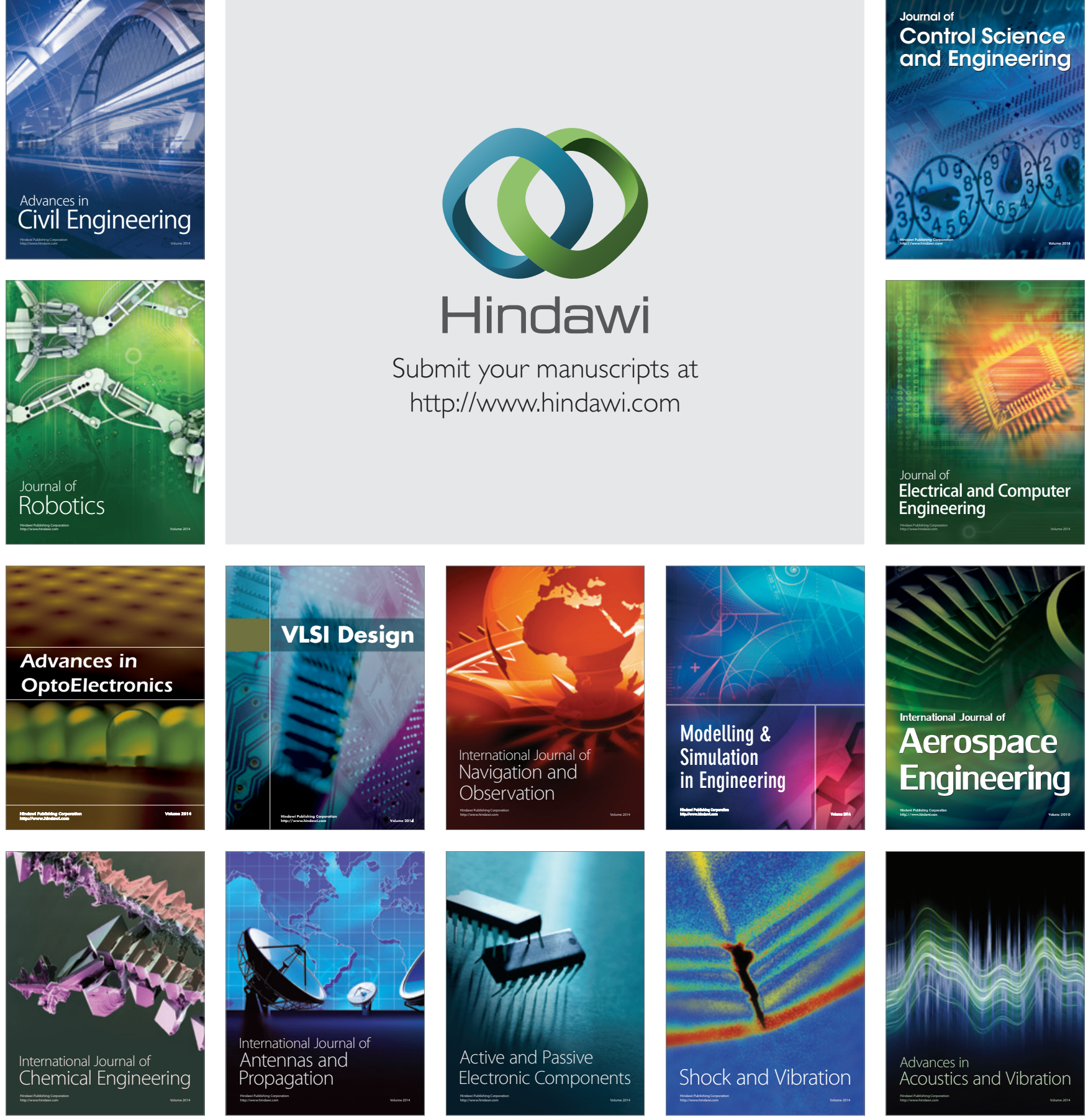